\title{
Oral Candidiasis
}

National Cancer Institute

\section{Source}

National Cancer Institute. Oral Candidiasis. NCI Thesaurus. Code C28137.

Infection of the mucosal lining of the mouth with the fungus Candida albicans. 